\title{
Anderson localization of entangled photons in an integrated quantum walk
}

\author{
Andrea Crespi, ${ }^{1,2}$ Roberto Osellame, ${ }^{1,2, \text { f }}$ Roberta Ramponi, ${ }^{1,2}$ Vittorio Giovannetti, ${ }^{3}$ Rosario \\ Fazio, ${ }^{3}$ Linda Sansoni, ${ }^{4}$ Francesco De Nicola, ${ }^{4}$ Fabio Sciarrino, ${ }^{4,5},{ }^{\dagger}$ and Paolo Mataloni ${ }^{4,5}$ \\ ${ }^{1}$ Istituto di Fotonica e Nanotecnologie, Consiglio Nazionale delle Ricerche \\ (IFN-CNR), Piazza Leonardo da Vinci, 32, I-20133 Milano, Italy \\ ${ }^{2}$ Dipartimento di Fisica, Politecnico di Milano, Piazza Leonardo da Vinci, 32, I-20133 Milano, Italy \\ ${ }^{3}$ NEST, Scuola Normale Superiore and Istituto di Nanoscienze - CNR, I-56126 Pisa, Italy \\ ${ }^{4}$ Dipartimento di Fisica, Sapienza Università di Roma, Piazzale Aldo Moro, 5, I-00185 Roma, Italy \\ ${ }^{5}$ Istituto Nazionale di Ottica, Consiglio Nazionale delle Ricerche (INO-CNR), Largo Enrico Fermi, 6, I-50125 Firenze, Italy
}

Waves fail to propagate in random media. First predicted for quantum particles in the presence of a disordered potential, Anderson localization has been observed also in classical acoustics, electromagnetism and optics. Here, for the first time, we report the observation of Anderson localization of pairs of entangled photons in a two-particle discrete quantum walk affected by position dependent disorder. A quantum walk on a disordered lattice is realized by an integrated array of interferometers fabricated in glass by femtosecond laser writing. A novel technique is used to introduce a controlled phase shift into each unit mesh of the network. Polarization entanglement is exploited to simulate the different symmetries of the two-walker system. We are thus able to experimentally investigate the genuine effect of (bosonic and fermionic) statistics in the absence of interaction between the particles. We will show how different types of randomness and the symmetry of the wave-function affect the localization of the entangled walkers.

In 1958 P.W. Anderson [1] predicted that the wavefunction of a quantum particle can be localized in the presence of a static disordered potential. As a consequence of this mechanism it is expected that particle and energy transport through a disordered medium should be strongly suppressed and that an initially localized wave packet should not spread out with time. After more than fifty years from its discovery Anderson localization is still widely studied and it has pervaded many different areas of physics ranging from condensed matter and cold atomic physics to wave dynamics and quantum chaos [2]. This phenomenon emerges quite generically in the behavior of waves in complex media, and it has been experimentally observed in a variety of different systems: BoseEinsten condensates [3, 4, light in semiconductor powders [5] and photonic lattices [6] 8, single photons in bulk optics 9] and in fiber loops [10, microwaves in strongly scattering samples [11, besides ultrasound waves in a three-dimensional elastic system [12].

\footnotetext{
*Electronic address: roberto.osellame@polimi.it
}

${ }^{\dagger}$ Electronic address: fabio.sciarrino@uniroma1.it

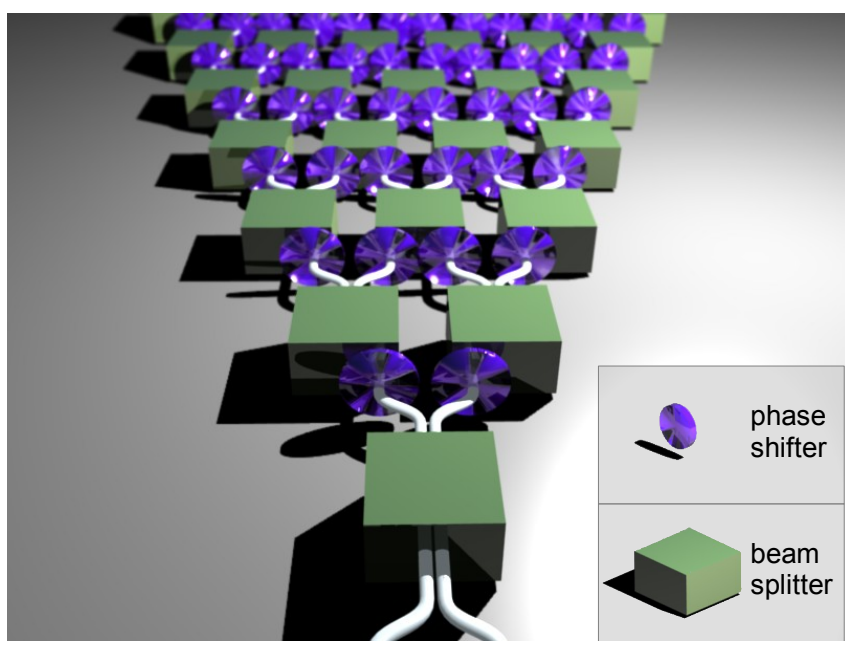

FIG. 1: Concept scheme of cascaded beam splitters to implement photonic quantum walks. Disorder is introduced by phase shifters placed at each beam splitter's output port, before entering the next one.

Anderson localization is a single-particle process which arises from the destructive interference among different scattering paths. Nevertheless, even in the absence of a direct interaction between particles, pure quantum correlations [13] are expected to influence in a non-trivial way the underlying localization dynamics [14-17]. By taking advantage of the perfect phase stability provided by miniaturized integrated waveguide circuits [18], we experimentally simulate a quantum walk of a two-photon polarization-entangled state in a disordered medium. We are thus able, through a mapping derived in Ref. 14, to test the localization of a pair of non interacting particles obeying bosonic/fermionic statistics 19.

A quantum walk (QW) $[20$ is an extension of the classical random walk, where the walker goes back and forth along a line and the direction at each step depends on the result of a fair coin flip. At the quantum level, interference and superposition phenomena lead to a non-classical behavior of the walker giving rise to new interesting effects, which can be harnessed to exponentially speed up search algorithms [21] and to realize universal quantum computation 22]. Besides, QWs have also been proposed to analyze energy transport in biological systems [23, 24]. Different experimental implementations of single-particle 

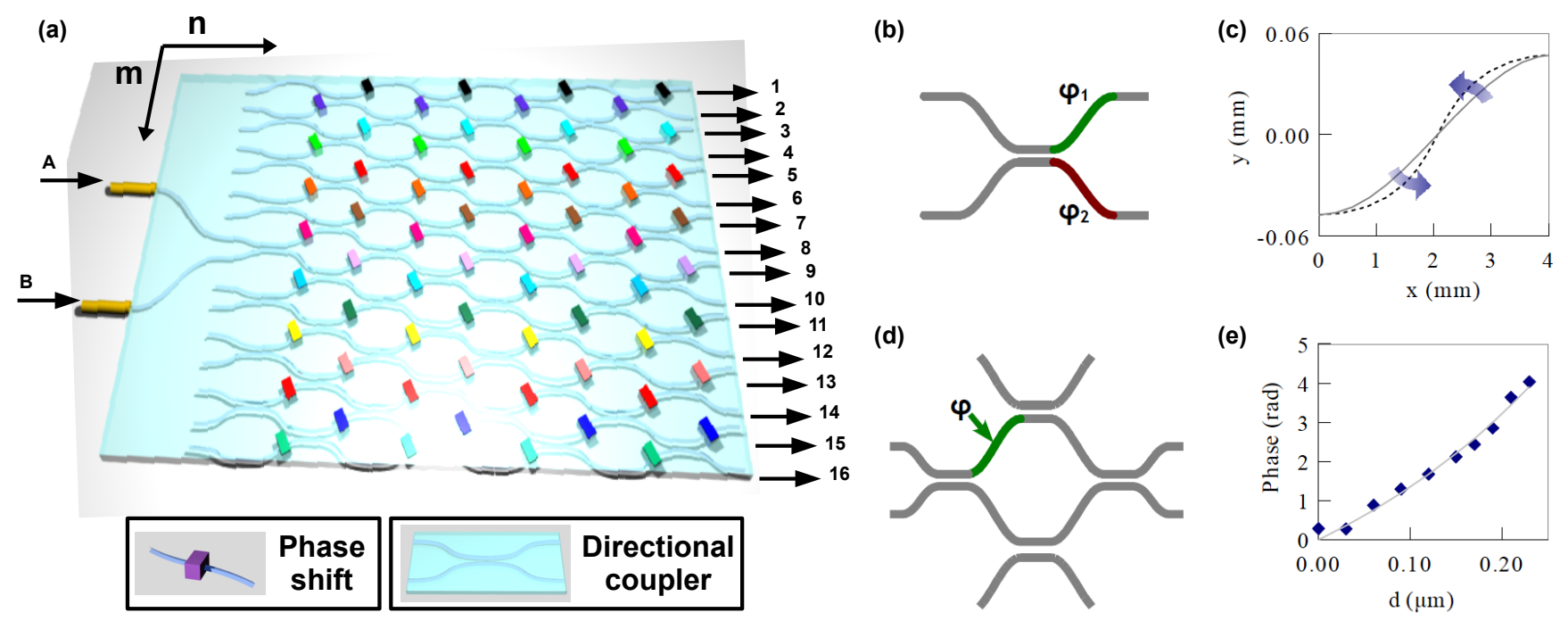

FIG. 2: (a) Scheme of the network of directional couplers implementing a 8-step quantum walk with static disorder. Different colors stand for different phase shifts. (b) Controlled deformation of either of the two S-bent waveguides at the output of each directional coupler extends the optical path and is equivalent to the application of a phase shifter. (c) The deformation is given by a non-linear coordinate transformation, which is function of a deformation coefficient $d$ (see Supplementary Information). The graph shows the undeformed S-bend (solid line), together with a deformed one (dashed). (d) Schematic of the Mach-Zehnder structure, representing the unit cell of the directional couplers network, fabricated for calibrating the phase shift induced by the deformation. (e) Phase shift induced by the deformation: theoretical curve calculated from the nominal geometric deformation (solid line), and experimental measurements (diamonds).

quantum walks were performed with trapped atoms [25], ions [26, 27, energy levels in NMR schemes [28, photons in waveguide structures 229, in bulk optics [9, 30, and in a fiber loop configuration [10, 31, 32. Very recently quantum walks of two identical photons have been performed only in ordered structures [18, 33, 34.

A physical realization of a discrete $\mathrm{QW}$ can be provided by photons passing through a cascade of balanced beam splitters (BSs) arranged in a network of Mach-Zehnder (MZ) interferometers as shown in Figure 1. Here each BS implements simultaneously the quantum coin operation, i.e. the choice of the direction the particle will move in, and the step operator, which shifts the walker in the direction fixed by the quantum coin state (the time evolution being simulated stroboscopically) [18. Accordingly every output of a BS of the network corresponds to a given point in the space-time of the $\mathrm{QW}$, the horizontal rows of the setup representing different time steps. In this scenario, disorder can be added in the QW evolution by simply introducing (randomly selected) phase shifts between the MZ interferometers paths (see Fig. 1). In particular the time-independent, static, disorder needed to enforce Anderson localization on the photonic walker, is obtained by fixing the same phases for all the MZs which correspond to the same lattice site. Making sure that response of the device is polarization independent, the localization of a non interacting entangled pair can now be studied by injecting in two different ports of the device -namely $A$ and $B$ in Fig $2 \mathrm{a}-\mathrm{a}$ twophoton entangled-polarized state generated via spontaneous parametric down-conversion. The above approach would be extremely hard to implement with bulk optics mainly because of size and of very challenging stability issues. However in the last few years integrated quantum photonics proved to be a highly promising experimental platform for quantum information science [36. Recently, integrated waveguide circuits have been employed for quantum applications, in order to realize twoqubit gates [35, 37 39], quantum algorithms [40], quantum walk on a chip [18, 33, 41] and enhanced quantum sensitivity in phase-controlled interferometers 42 44]. In order to observe Anderson localization for polarization entangled photons an important step forward is required in the available experimental platform. Our setup, for the first time, integrate all the necessary ingredients to this aim: polarization independent elements, interferometric structures, together with a proven capability to implement suitable phase shift in different points of the QW circuit. Furthermore, in order to get a convincing evidence of localization it has been necessary to observe the experimental simulation for quantum walk of different steps. We were able to realize up to eight-step QW circuits affected by a controlled disorder, thus integrating tens of BSs on the same chip.

In our experiment the setup of Figure 1 has been realized by using integrated waveguide circuits, as in Figure $2 \mathrm{a}$, where BS elements are replaced by directional couplers. The discrete $m$-axis indicates the different sites of the QW, while the discrete $n$-axis identifies the different time steps. The integrated waveguide circuits have been fabricated by femtosecond laser writing technology [45, 46]. This technology exploits nonlinear absorp- 
tion of femtosecond pulses, focused below the surface of a transparent dielectric substrate, to obtain a permanent and localized refractive index increase. Translation of the sample under the laser beam along the desired path enables the fabrication of optical waveguide circuits with arbitrary three-dimensional geometries. In order to obtain a totally polarization independent behavior, the 3-dimensional geometry detailed in Ref. [18] has been adopted (these devices are known to allow the propagation of polarization entangled states [35]). The phase shifters are implemented by deforming one of the S-bent waveguides at the output of each directional coupler (green or red segments in Figure 2b), in order to stretch the optical path. The phase shift $[-\pi, \pi]$ in each MZ cell is implemented by lengthening the optical path in the green segment to introduce a $[0, \pi]$ phase shift, while the complementary range $[-\pi, 0]$ is achieved by lengthening the red segment. In this way, smaller deformations, always of the same kind (lengthening of the path), are capable to provide the full range of phase shifts.

Figure 2p shows both an undeformed and a deformed S-bend.

\begin{tabular}{|c|c|c|}
\hline \multicolumn{3}{|c|}{ ORDERED } \\
\hline Steps & Input $A$ & Input $B$ \\
\hline 4 & $0.991 \pm 0.002$ & $0.992 \pm 0.002$ \\
\hline 6 & $0.994 \pm 0.003$ & $0.987 \pm 0.003$ \\
\hline 8 & $0.951 \pm 0.004$ & $0.946 \pm 0.005$ \\
\hline \multicolumn{3}{|c|}{ STATIC } \\
\hline Steps & Input $A$ & Input $B$ \\
\hline 4 & $0.980 \pm 0.003$ & $0.976 \pm 0.002$ \\
\hline 6 & $0.985 \pm 0.002$ & $0.976 \pm 0.003$ \\
\hline 8 & $0.938 \pm 0.004$ & $0.957 \pm 0.004$ \\
\hline
\end{tabular}

TABLE I: Similarities for single particle QW distributions. The values are calculated as mean average on distributions of single-photon in different polarization states.

To test our technique and calibrate the achieved phase shift as a function of the imposed deformation $d$ (see Supplementary Information for a detailed definition) several MZ interferometers were fabricated with the design of Figure 2 d), reproducing exactly the unit cell of the QW network. Each interferometer has one S-bend (the one colored in the figure) deformed with a different value of d. Laser light at $\lambda=806 \mathrm{~nm}$ wavelength was injected in the interferometers and the induced phase shift was then retrieved from the measured light distribution at the output. Figure 2 e) reports the experimentally measured phase shifts as a function of the deformation parameter $d$. The experimental points are in good agreement with the phase shift predicted by evaluating numerically the geometric lengthening $\Delta l$ of the deformed S-bend $\phi_{\text {theo }}=\frac{2 \pi}{\lambda} \Delta l$.

We implemented a lattice with static disorder by imposing the same phase shift to the MZ cells correspond- ing to a fixed site of the QW line as in Fig. $2 \mathrm{a}\left(\phi_{m, n}=\right.$ $\left.\phi_{m}, \forall n\right)$. QW circuits composed by 4,6 and 8 step affected by static disorder were realized in a way that the 4-step phase pattern was embedded within the 6-step phase pattern and, in turn, this was embedded within the 8-step one.

Another set of 4, 6 and 8 step ordered, i.e. with perfectly symmetric MZ cells, QW circuits was realized and compared with the corresponding disordered one.

First of all we measured the single particle distributions (see Supplementary Information) in order to demonstrate the polarization insensitivity of the integrated QWs. We repeated this measurement by injecting single photon states with different polarizations. The measured distributions exhibit very similar behaviors. We compared the obtained results with the expected ones by calculating the similarity defined as $S=$ $\left(\sum_{i, j} \sqrt{D_{i j} D_{i, j}^{\prime}}\right)^{2} / \sum_{i, j} D_{i j} \sum_{i, j} D_{i j}^{\prime}$, which is a generalization of the classical fidelity between two distributions $D$ and $D^{\prime}$. The obtained values are reported in Table I for the ordered QW circuits and for QWs with static disorder. These high values and low deviations highlight once more the fabrication control and polarization insensitivity of our integrated devices.

As a second step we carried out the investigation of two-particle QWs. The investigation of Anderson localization for bosonic and fermionic particles was realized by adopting the complete experimental setup described in details in the Supplementary Information. As mentioned above, we exploited polarization entanglement to simulate bosonic and fermionic statistics. To this aim polarization-entangled photon pairs, generated via spontaneous parametric down-conversion, were simultaneously injected into arms $A$ and $B$ of the 4,6 and 8 steps QW circuits to observe the progressive quench of photon propagation in disordered QWs (Fig. 2a). By setting the phase $\phi$ of the state $\frac{1}{\sqrt{2}}\left[|H\rangle_{A}|V\rangle_{B}+e^{i \phi}|V\rangle_{A}|H\rangle_{B}\right]$, bosonic $(\phi=0)$ or fermionic $(\phi=\pi)$ QWs were observed. In Figure 3 we show how entangled photons localize in the presence of a random static potential by plotting the joint probability $P_{j, k}$ of detecting one particle in the output port $j$ and the other in the output port $k$ (the probability being determined by collecting events independently from the photon polarization). The different panels compare the ordered and disordered cases in the case of symmetric and antisymmetric wave-functions. We report also the case of single photons which are reconstructed by tracing out the position of one of the particles of the entangled pair. While in the case of an ordered system the walkers spread on with the increasing number of steps, Anderson localization implies that the wave-packets will remain localized around the central sites irrespectively of the number of steps. This is indeed what we observed. The difference between the ordered and disordered case is most evident for the 8-step QW, compare the panels (g) and $(\mathrm{h})$ with $(\mathrm{r})$ and $(\mathrm{s})$ in Figure 3 . The agreement of the experimental data with the theoretical predictions, 
ORDERED
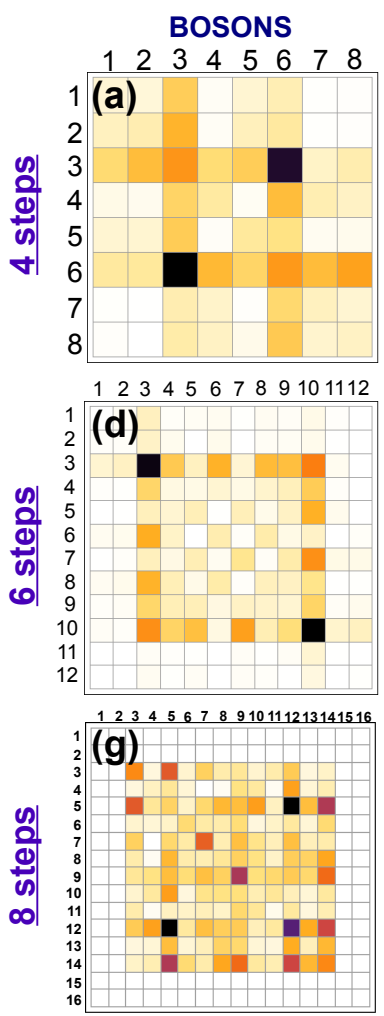

FERMIONS

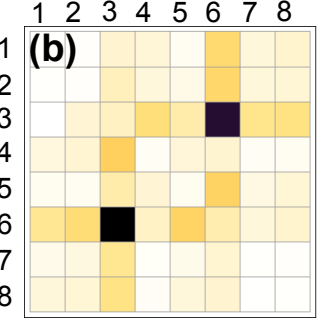

123456789101112
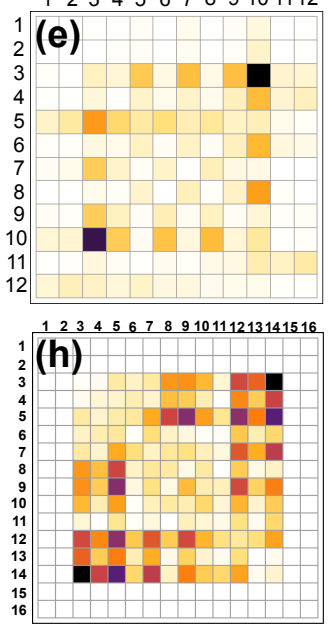

\section{STATIC}

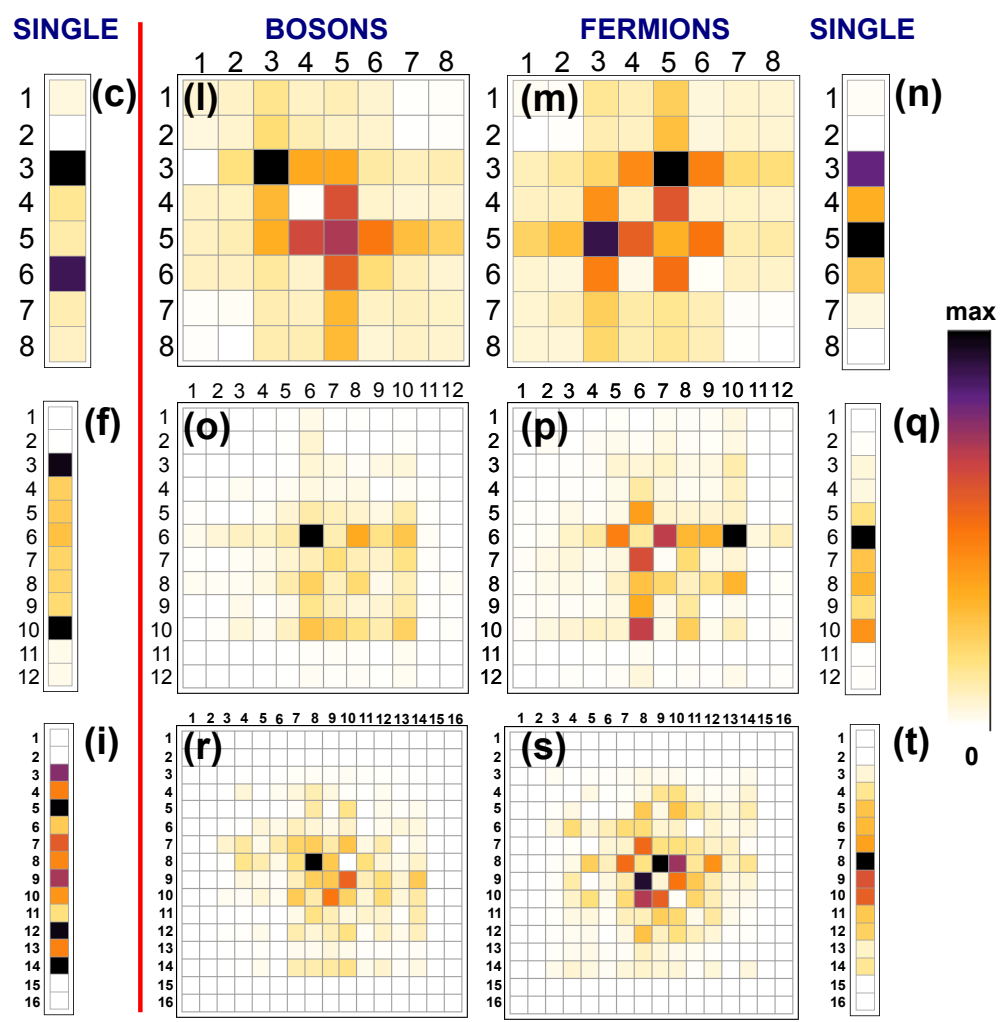

FIG. 3: Experimental results of single- and two-photon distributions for bosons and fermions in an ordered QW (a-i) and in a QW in presence of static disorder (l-t). Single-particle density distributions have been computed by tracing out the position of one of the particles (summing over the columns of the two-photon probability distribution matrices).

\begin{tabular}{|c|c|c|c|c|c|c|c|}
\hline \multicolumn{4}{|c|}{ ORDERED } & \multicolumn{4}{c|}{ STATIC } \\
\cline { 1 - 7 } Steps & Bosons & Fermions & Single & Steps & Bosons & Fermions & Single \\
\hline 4 & $0.946 \pm 0.003$ & $0.914 \pm 0.003$ & $0.996 \pm 0.001$ & & $0.918 \pm 0.003$ & $0.902 \pm 0.003$ & $0.993 \pm 0.001$ \\
\hline 6 & $0.940 \pm 0.003$ & $0.851 \pm 0.003$ & $0.997 \pm 0.001$ & & $0.890 \pm 0.006$ & $0.903 \pm 0.004$ & $0.985 \pm 0.002$ \\
\hline 8 & $0.768 \pm 0.006$ & $0.780 \pm 0.007$ & $0.934 \pm 0.004$ & 8 & $0.803 \pm 0.004$ & $0.785 \pm 0.004$ & $0.947 \pm 0.002$ \\
\hline
\end{tabular}

TABLE II: Similarities between the experimental distributions of Fig. 3 and the expected ones. Uncertainties arise from the Poisson distribution of counting statistics.

again quantified by the similarities, is reported in Table II] In the ordered case $\mathcal{S}$ is slightly worse for the 8-step QW. This discrepancy, due to some unavoidable uncertainty in BSs realization is milder in the disordered case. Here, as expected, additional phase-shift to the "intentionally-chosen" random one will have less effect due to localization.

The entangled pairs localize in a manner which depends on their statistics. A more quantitative estimate of the difference in the localization properties of entangled photons may be obtained by looking at the variance of the two-photon mean position $x_{M}=(j+k) / 2$ associated with the probability distributions of Figure 3 as a function of the number of steps. This is shown in Figure 4 a,b. While for the ordered case the variance grows quadratically with the number of steps $n$, it is weakly dependent on $n$ in the disordered case, indicating that the system tends towards localization. The numerical simulation, performed by considering discrete time QWs with static disorder shows that localization starts even with a relatively small number of steps, giving rise to a fully localized state (corresponding to a variance not varying with $n$ ) typically for $n \sim 100$. However, such number of steps is currently out-of-reach for any technological platform. The clear difference observed in our experiment between ordered and disordered QWs is a strong evidence of the onset of Anderson localization for a small number of steps.

In addition we observe a very interesting feature emerging from the data, both in Fig. 3 and in Fig. 4 , further 

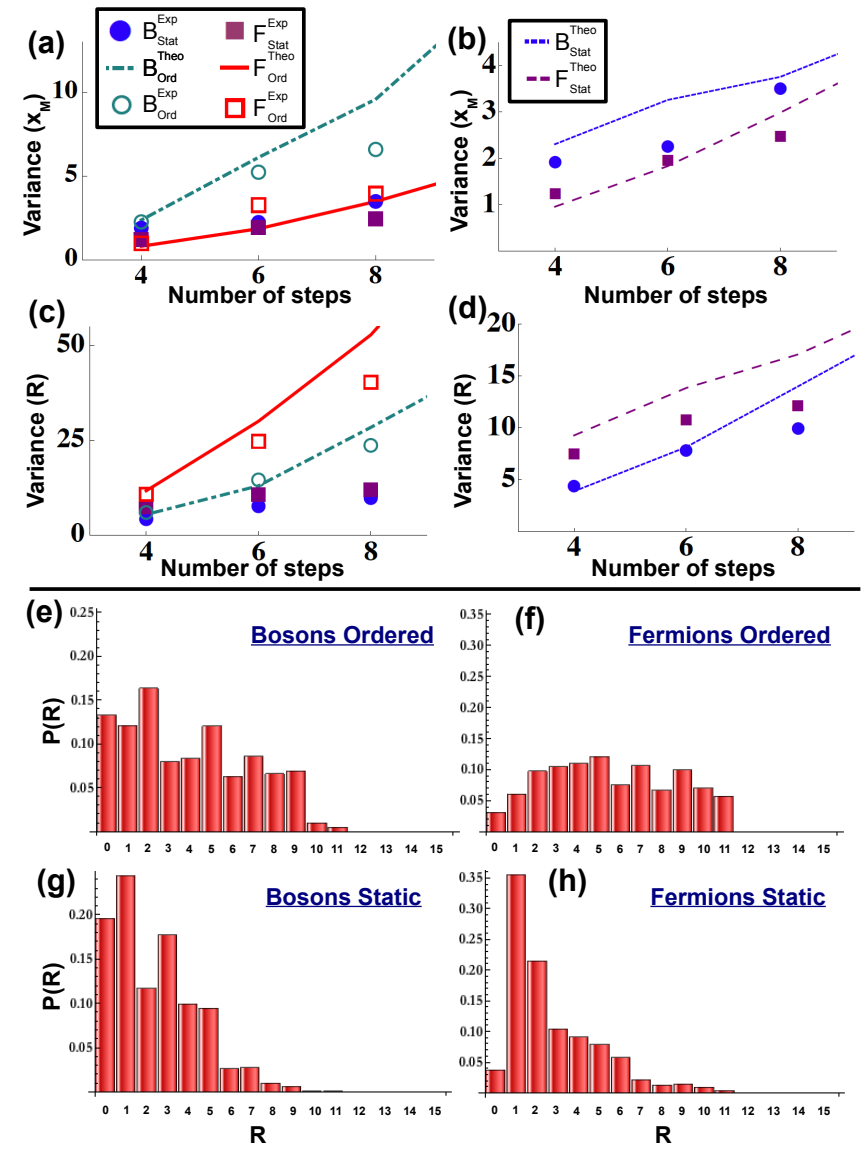

FIG. 4: (a-b) Variance of the two photon mean position $x_{M}=(j+k) / 2$ and $(\mathrm{c}, \mathrm{d})$ of the relative distance $R=|i-k|$ shown in function of the number of steps. Experimental results are reported for bosons (circles) and fermions (squares). Empty markers refer to ordered QWs, whereas filled ones correspond to QWs with static disorder. In panels a,b dot-dashed and solid lines represent theoretical behaviors for bosons and fermions in the ordered case while in panels c,d dotted and dashed lines correspond to theory for bosons and fermions in presence of static disorder, respectively. Error bars are smaller than the spot size. (e-h) Probability distributions of the relative distance $R$ for bosonic (left) and fermionic (right) two-photon 8-step QWs in the case of a ordered QW (e-f) and in presence of position-dependent disorder $(\mathrm{g}-\mathrm{h})$.

evidencing the different behaviour between bosons and fermions. By looking at the behavior of $x_{M}$, fermions localize more than bosons. Antisymmetry does help localization. This fact that may sound counterintuitive can be understood (see the Supplementary Information) by looking at the sign of the interference term on the variance of $x_{M}$. An opposite behavior is observed for the distribution of the relative distance $R=|j-k|$ between the two particles. Because of the Pauli exclusion principle, the average distance between the particles in the fermionic case is larger than in the bosonic case (where they tend to bunch). This is exactly what we observe by looking at the variance of the distribution of the relative distances (Fig. 44,d), which follows from the probability distribution $P(R)$. The different experimental distributions of the distance between the particles, obtained in both the ordered and static-disordered case, and the different behaviour of bosons and fermions are shown in Fig. 4h-h.

So far we discussed the case of static disorder, however different types of disorder affect differently the dynamics of the entangled pairs. Since our technology is capable of implementing arbitrary phase maps in the QWs, a lattice with dynamic disorder was produced applying the same phase shifts to MZs belonging to a fixed step of the walk $\left(\phi_{m, n}=\phi_{n}, \forall m\right)$, as in Figure 3b of the Supplementary Information. Experimental single- and two-photon output distributions are summarized in Figure 5 a,b for the bosonic and fermionic case separately. The effect of a fully space-correlated dynamic randomness simulates the effect of an external classical environment. In this case one can show that the limiting distribution is a binomial centered in the middle of the spatial axis and with width growing linearly with the square root of the number of steps. Thus the system undergoes a diffusion process in which the propagation becomes equivalent to a purely classical random walk. The data of Figure 5 a-c indeed confirm that in the case of dynamic disorder the walkers spread more easily on the BS tree with respect to the static case, but spread less with respect to the ballistic diffusion of the ordered case.

The last scenario we considered is the case of both space- and time-dependent disorder, which we will call fluctuating disorder, realized with random phase shifts $\phi_{m, n}$ over the entire MZ network (see Fig. 3c of the Supplementary Information). In this configuration the diffusion process leads to a speckle pattern for the two-walkers wavefunction (Figure 5 $\mathrm{d}-\mathrm{f}$ ). This case shows that the interaction with an external classical environment quenches the localization effect that would be induced by a lattice with static disorder.

Let us note that the experiments are performed on a single phase map realization of each disorder. Although there are still features that are linked to the particular choice of the (randomly picked) phase maps, the number of beam splitters is large enough to allow the clear observation of the differences between ballistic, diffusive and localized regimes.

We reported on the experimental realization of a quantum simulator based on discrete quantum walks of entangled particles in integrated photonic circuits. By properly engineering the phase shifts at the output ports of the BSs and by changing the number of QW steps, we were able to follow in real time the evolution towards Anderson localization. The symmetry of the total wavefunction (Fermi- or Bose-like) clearly affects the localization properties. Fermi statistics helps localization despite the fermions tendency to antibunching. The quantum simulation we performed will help to ascertain the efficiency of quantum algorithms with entangled particles on realistic quantum walks. The capability of our technology to implement arbitrary phase maps in QWs paves the way 


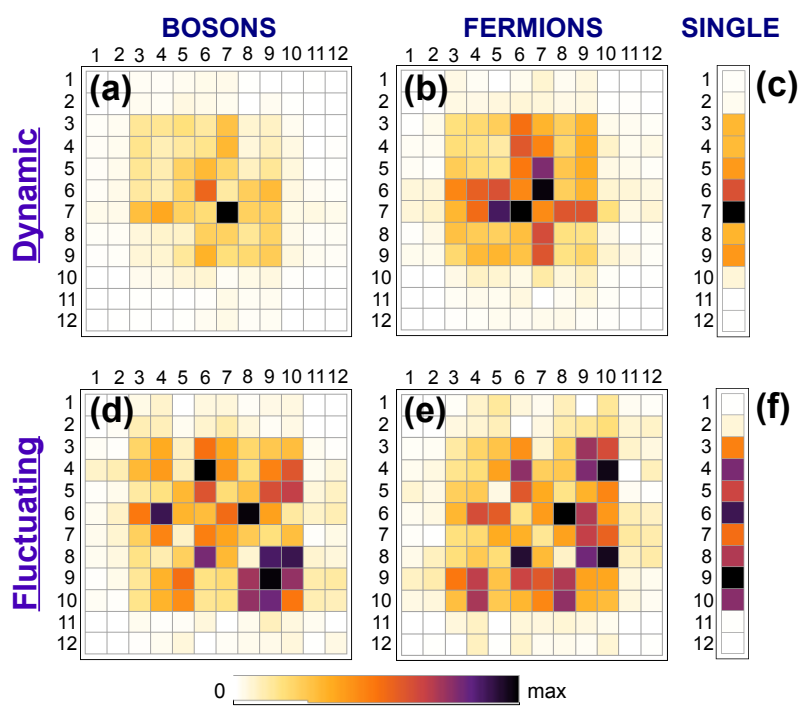

FIG. 5: Experimental results: single- and two-particle distributions for a 6-step QW in presence of dynamic (a-c) and fluctuating (d-f) disorder. Single particle distributions have been computed by tracing out the position of one of the particles (summing over the columns of the probability distribution matrices) The similarities for bosons, fermions and single particle with the expected distributions are respectively $\mathcal{S}_{\text {Bos }}^{D}=0.871 \pm 0.004, \mathcal{S}_{\text {Fer }}^{D}=0.802 \pm 0.006$ and $\mathcal{S}_{\text {Sing }}^{D}=0.975 \pm 0.003$ for the QW circuit with dynamic disorder and $\mathcal{S}_{\text {Bos }}^{F}=0.921 \pm 0.004, \mathcal{S}_{\text {Fer }}^{F}=0.852 \pm 0.003$ and $\mathcal{S}_{\text {Sing }}^{F}=0.991 \pm 0.002$ for the chip with fluctuating disorder. to the experimental quantum simulation of the quantum dynamics of multi-particle correlated systems and its ramifications towards the implementation of realistic universal quantum computation with quantum walks.

This project was supported by FIRB- Futuro in Ricerca HYTEQ, PRIN 2009, IP-SOLID and ERCStarting Grant: 3D-QUEST.

L.S, F.D.N., F.S., P.M., A.C., R.O. and R.R. conceived the experimental approach for the simulation of the Anderson localization. A.C., R.O., and R.R. fabricated the integrated devices. L.S., F.D.N., F.S., and P.M. carried out the experiments. V.G. and R.F. contributed to the theoretical analysis on how statistics influence the localization. All the authors discussed the experimental implementation and results and contributed to writing the paper.
[1] P. Anderson, Absence of diffusion in certain random lattices, Physical Review 109, 1492 (1958).

[2] E. Abrahams, 50 years of Anderson localization, World Scientific, (Singapore) (2010).

[3] G. Roati, C. D'Errico, L. Fallani, M. Fattori, C. Fort, M. Zaccanti, G. Modugno, M. Modugno, and M. Inguscio, Anderson localization of a non-interacting boseñeinstein condensate, Nature (London) 453, 895 (2008).

[4] J. Billy, V. Josse, Z. Zuo, A. Bernard, B. Hambrecht, P. Lugan, D. ClÈment, L. Sanchez-Palencia, P. Bouyer, and A. Aspect, Direct observation of anderson localization of matter waves in a controlled disorder, Nature (London) 453, 891 (2008).

[5] D. S. Wiersma, P. Bartolini, A. Lagendijk, and R. Righini, Localization of light in a disordered medium, Nature (London) 390, 671 (1997).

[6] T. Pertsch, J. K. U. Peschel, K. Schuster, H. Bartelt, S. Nolte, A. Tünnermann, and F. Lederer, Nonlinearity and disorder in fiber arrays, Physical Review Letters 93, 053901 (2004).

[7] T. Schwartz, G. Bartal, S. Fishman, and M. Segev, Transport and anderson localization in disordered twodimensional photonic lattices, Nature (London) 466, 52 (2007).

[8] Y. Lahini, A. Avidan, F. Pozzi, M. Sorel, R. Morandotti, D. N. Christodoulides, and Y. Silberberg, Anderson lo- calization and nonlinearity in one-dimensional disordered photonic lattices, Physical Review Letters 100, 013906 (2008).

[9] M. A. Broome, A. Fedrizzi, B. P. Lanyon, I. Kassal, A. Aspuru-Guzik, and A. G. White, Discrete singlephoton quantum walks with tunable decoherence, Physical Review Letters 104, 153602 (2010).

[10] A. Schreiber, K. N. Cassemiro, V. Potocek, A. Gabris, P. J. Mosley, I. Jex, and C. Silberhorn, Decoherence and disorder in quantum walks: From ballistic spread to localization, Physical Review Letters 106, 180403 (2011).

[11] M. Störzer, P. Gross, C. M. Aegerter, and G. Maret, Observation of the critical regime near anderson localization of light, Physical Review Letters 96, 063904 (2006).

[12] H. Hu, A. Strybulevych, J. H. Page, S. E. Skipetrov, and B. A. van Tiggelen, Localization of ultrasound in a three-dimensional elastic network, Nature Physics 4, 945 (2008).

[13] These can be either in the form of quantum entanglement of the initial state or in the form of the quantum statistics of the involved particles.

[14] Y. Omar, N. Paunković, and L. S. S. Bose, Quantum walk on a line with two entangled particles, Physical Review A 74, 042304 (2006).

[15] C. Beenakker, J. Venderbos, and M. van Exter, Twophoton speckle as a probe of multi-dimensional entanglement, Physical Review Letters 102, 193601 (2009).

[16] Y. Lahini, Y. Bromberg, D. N. Christodoulides, and 
Y. Silberberg, Quantum correlations in two-particle anderson localization, Physical Review Letters 105, 163905 (2010).

[17] Y. Bromberg, Y. Lahini, M. R, and Y. Silberberg, Quantum and classical correlations in waveguide lattices, Physical Review Letters 102, 253904 (2009).

[18] L. Sansoni, F. Sciarrino, G. Vallone, P. Mataloni, A. Crespi, R. Ramponi, and R. Osellame, Two-particle bosonic-fermionic quantum walk via integrated photonics, Physical Review Letters 108, 010502 (2012).

[19] This situation may also be contrasted with the localization of pairs of interacting particles theoretically considered in D. Shepelyansky, Coherent propagation of two interacting particles in a random potential, Physical Review Letters 73, 2607 (1994).

[20] J. Kempe, Quantum random walks - an introductory overview, Contemporary Physics 44, 307 (2003).

[21] V. Potocek, A. Gabris, T. Kiss, and I. Jex, Optimized quantum random-walk search algorithms on the hypercube, Physical Review A 79, 012325 (2009).

[22] A. M. Childs, Universal computation by quantum walk, Physical Review Letters 102, 180501 (2009).

[23] M. Mohseni, P. Rebentrost, S. Lloyd, and A. AspuruGuzik, Environment-assisted quantum walks in photosynthetic energy transfer, The Journal of Chemical Physics 129, 174106 (2008).

[24] P. Rebentrost, M. Mohseni, I. Kassal, S. Lloyd, and A. Aspuru-Guzik, Environment-assisted quantum transport, New Journal of Physics 11, 033003 (2009).

[25] M. Karski, L. Forster, J.-M. Choi, A. Steffen, W. Alt, D. Meschede, and A. Widera, Quantum walk in position space with single optically trapped atoms, Science 325, 174 (2009).

[26] H. Schmitz, R. Matjeschk, C. Schneider, J. Glueckert, M. Enderlein, T. Huber, and T. Schaetz, Quantum walk of a trapped ion in phase space, Physical Review Letters 103, 090504 (2009).

[27] F. Zahringer, G. Kirchmair, R. Gerritsma, E. Solano, R. Blatt, , and C. F. Roos, Realization of a quantum walk with one and two trapped ions, Physical Review Letters 104, 100503 (2010).

[28] C. A. Ryan, M. Laforest, J. C. Boileau, and R. Laflamme, Experimental implementation of a discrete-time quantum random walk on an nmr quantum-information processor, Physical Review A 72, 062317 (2005).

[29] H. B. Perets, Y. Lahini, F. Pozzi, M. Sorel, R. Morandotti, and Y. Silberberg, Realization of quantum walks with negligible decoherence in waveguide lattices, Physical Review Letters 100, 170506 (2008).

[30] T. Kitagawa, M. A. Broome, A. Fedrizzi, M. S. Rudner, E. Berg, I. Kassal, A. Aspuru-Guzik, E. Demler, and A. G. White, Observation of topologically protected bound states in photonic quantum walks, Nature Communications 3, 882 (2012).

[31] A. Schreiber, K. N. Cassemiro, V. Potocek, A. Gabris, P. J. Mosley, E. Andersson, I. Jex, and C. Silberhorn, Photons walking the line: A quantum walk with adjustable coin operations, Physical Review Letters 104, 050502 (2010).

[32] A. Schreiber, A. Gábris, P. P. Rohde, K. Laiho, M. Ste- fank, V. Potocek, C. Hamilton, I. Jex, and C. Silberhorn, A $2 d$ quantum walk simulation of two-particle dynamics, Science 336, 55 (2012).

[33] A. Peruzzo, M. Lobino, J. C. F. Matthews, N. Matsuda, A. Politi, K. Poulios, X.-Q. Zhou, Y. Lahini, N. Ismail, K. Worhoff, Y. Bromberg, Y. Silberberg, M. G. Thompson, and J. L. O'Brien, Quantum walks of correlated photons, Science 329, 1500 (2010).

[34] J. O. Owens, M. A. Broome, D. N. Biggerstaff, M. E. Goggin, A. Fedrizzi, T. Linjordet, M. Ams, G. D. Marshall, J. Twamley, M. J. Withford, and A. G. White, Two-photon quantum walks in an elliptical direct-write waveguide array, New Journal of Physics 13, 075003 (2011), arXiv:1103.0604v1 (2011).

[35] L. Sansoni, F. Sciarrino, G. Vallone, P. Mataloni, A. Crespi, R. Ramponi, and R. Osellame, Polarization entangled state measurement on a chip, Physical Review Letters 105, 200503 (2010).

[36] J. L. O'Brien, A. Furusawa, and J. Vuckovic, Photonic quantum technologies, Nature Photonics 3, 687 (2009).

[37] A. Politi, M. J. Cryan, J. G. Rarity, S. Yu, and J. L. O'Brien, Silica-on-silicon waveguide quantum circuits, Science 320, 646 (2008).

[38] A. Laing, A. Peruzzo, A. Politi, M. R. Verde, M. Halder, T. C. Ralph, M. G. Thompson, and J. L. O'Brien, Highfidelity operation of quantum photonic circuits, Applied Physics Letters 97, 211108 (2010).

[39] A. Crespi, R. Ramponi, R. Osellame, L. Sansoni, I. Bongioanni, F. Sciarrino, G. Vallone, and P. Mataloni, Integrated photonic quantum gates for polarization qubits, Nature Communications 2, 566 (2011).

[40] A. Politi, J. C. F. Matthews, and J. L. O'Brien, Shor's quantum factoring algorithm on a photonic chip, Science 325, 1221 (2009).

[41] J. C. F. Matthews, K. Poulios, J. D. A. Meinecke, A. Politi, A. Peruzzo, N. Ismail, K. Worhoff, M. G. Thompson, and J. L. O'Brien, Simulating quantum statistics with entangled photons: a continuous transition from bosons to fermions (2011), arXiv: quantph71106.1166.

[42] B. J. Smith, D. Kundys, N. Thomas-Peter, P. G. R. Smith, and I. A.Walmsley, Phase-controlled integrated photonic quantum circuits, Optics Express 17, 13516 (2009).

[43] J. C. F. Matthews, A. Politi, A. Stefanov, and J. L. O'Brien, Manipulation of multiphoton entanglement in waveguide quantum circuits, Nature Photonics 3, 346 (2009).

[44] A. Crespi, M. Lobino, J. C. F. Matthews, A. Politi, C. R. Neal, R. Ramponi, R. Osellame, and J. L. O???Brien, Measuring protein concentration with entangled photons, Applied Physics Letters 100, 233704 (2012).

[45] R. R. Gattass and E. Mazur, Femtosecond laser micromachining in transparent materials, Nature Photonics 2, 219 (2008).

[46] G. Della Valle, R. Osellame, and P. Laporta, Micromachining of photonic devices by femtosecond laser pulses, Journal of Optics A: Pure and Applied Optics 11, 049801 (2009). 